\title{
Performance of Large Intelligent Surface-enabled Cooperative Networks Over Nakagami- $m$ Channels
}

\author{
Madi Makin, Galymzhan Nauryzbayev, ${ }^{\circ}$ Sultangali Arzykulov, and Mohammad S. Hashmi \\ School of Engineering and Digital Sciences, Nazarbayev University, Nur-Sultan, Kazakhstan \\ ${ }^{\circ}$ Computer, Electrical, and Mathematical Sciences \& Engineering Division, King Abdullah University of Science \\ and Technology, Thuwal, Saudi Arabia \\ Email: \{madi.makin, galymzhan.nauryzbayev, mohammad.hashmi\}@nu.edu.kz, ${ }^{\circ}$ sultangali.arzykulov@kaust.edu.sa
}

\begin{abstract}
In this paper, we analyze the system performance of a large intelligent surface (LIS) enabled wireless system over Nakagami- $m$ channels. We derive closed-form expressions of the outage probability, ergodic capacity, and average bit error rate using different approximation methods, namely, central limit theorem (CLT), Gamma and generalized- $K\left(K_{\mathrm{G}}\right)$. The effects of a number of passive LIS elements $(N)$ and fading parameters on the system performance are examined. Results show that the Gamma and $K_{\mathrm{G}}$ approximations are precise given different values of $N$, while the CLT approximation's accuracy depends on the number of LIS elements implemented. Finally, analytical findings are validated by thorough Monte Carlo simulations.

Index Terms-Bit error rate (BER), cooperative communications, ergodic capacity, large intelligent surface (LIS), outage probability (OP).
\end{abstract}

\section{INTRODUCTION}

Large intelligent surfaces (LISs) have been proposed as a promising technology for the next generation of wireless networks. The LIS is a device consisting of a large number of reflective elements, with every element having a capability of controlling the phase and amplitude of incident signals [1]. So, with the proper phase-shift adjustment and signal alignment technique, the system performance at a receiver side can be improved significantly [2]. Due to its low energy consumption, cost-effectiveness, and high spectrum efficiency, LIS can become one of the solutions to overcome the fundamental challenges of fifth-generation (5G) and beyond wireless networks. With its increasing popularity, different LIS configurations have been studied, e.g., large intelligent meta-surfaces (LIM), reconfigurable intelligent surface (RIS), etc. [3], [4].

The performance of relay-aided and LIS-aided systems was compared in [5], where the results demonstrated that the latter obtains better performance than the former due to the large number of reflective elements deployed. The LIS/antenna (LISA) technology was studied in [6] while taking into account the basic characteristics and highlighting a variety of potential LISA-based applications. The authors in [7] investigated the beamforming optimization in RIS-aided systems under discrete phase-shift constraints and showed that one-bit shifters can achieve equal asymptotic power gain as continuous shifters do, although with constant power loss. Another application of the RIS/LIS-aided communications in non-orthogonal multiple access (NOMA) networks was studied in [8], [9]. It was noted that the direct channel between the source and destination nodes does not affect the system performance and thus can be neglected while providing a sufficient number of reflective elements at a relay node.

Most of the works mentioned above approximated the statistical distributions of channel links in the wireless networks with the help of a central limit theorem (CLT) using Gaussian distribution. Its accuracy depends on the number of reflective elements integrated; for example, a small number of RIS elements leads to in a considerable gap between the analytical and simulated results. Moreover, using the CLT, analytical expressions for the performance metrics, such as average symbol error probability, outage probability and achievable rate, of a RIS system were derived in [10]. The authors confirmed that the derived analytical expressions based on the CLT approximation become tighter to the simulated ones given a large number of reflective elements; therefore, it can be expected that these expressions would perform better for LIS-aided systems. Another type of approximation found to be more precise than the latter for RIS scenarios is a Gamma approximation [11]. To address the CLT limitation, the authors in [12] proposed an approach based on the sum of generalized $K$-distribution $\left(K_{\mathrm{G}}\right)$ random variables (RVs) to ensure the results tightness for the systems with a few reflective elements deployed. Their results showed that the $K_{\mathrm{G}}$ distribution can tightly approximate the RIS-aided system and perform better than the Gaussian approximation under Rayleigh fading.

Considering the aforementioned works, this letter examines the performance of a LIS-aided communication system over Nakagami- $m$ fading channels under three different approximations, i.e., Gaussian, Gamma and $K_{\mathrm{G}}$. First, closed-form expressions for outage performance (OP), ergodic capacity (EC), and average bit error rate (BER) are analytically derived for given approximations. Second, the accuracy and tightness of the obtained expressions are verified via Monte Carlo simulations. We show that the CLT approximation is not that accurate, even considering a large number of reflective elements, while Gamma and $K_{\mathrm{G}}$ methods are more attractive in terms of their accurateness for the considered LIS system. Through our analysis, we verify that LIS has a real potential of enhancing the system performance, and, by controlling the propagation environment, boosting the signal quality at the receiver. 


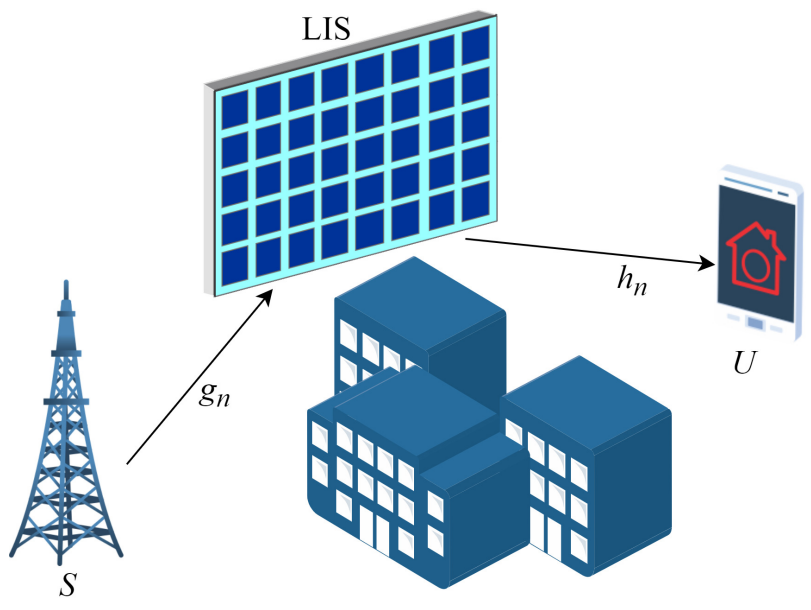

Fig. 1. A LIS-enabled wireless system.

\section{SyStem AND Channel Modeling}

\section{A. System Topology}

In this paper, we consider the signal transmission arising from the single-antenna source $(S)$ and designated to the user $(U)$ via LIS deployed with $N$ passive elements (see Fig. 1). It is assumed that, due to the dense urban environment, $S$ and $U$ do not have a direct line-of-sight (LoS) channel link. Moreover, the changes in an incident signal are considered to be in-phase, that is, the phases are controlled perfectly, as in [13], using a constructive signal accumulation at $U$. All channel links $\left(g_{n}\right.$ and $h_{n}$ ) are subject to a Nakagami- $m$ fading model and the channel envelopes are assumed to be independently but not necessarily identically distributed (i.n.i.d.).

\section{B. Transmission Protocol}

The signal received at $U$ can be written as

$$
y=\sqrt{P_{S}}\left(\sum_{n=1}^{N} g_{n} h_{n} d_{s r}^{-\frac{\tau}{2}} d_{r u}^{-\frac{\tau}{2}} \alpha_{n} e^{j \theta_{n}}\right) x+n,
$$

where $P_{S}, x$ and $n$ are the transmit power, message of $S$ and additive white Gaussian noise (AWGN) at a receiver node, with variance $N_{0}$, respectively. $d_{s r}$ and $d_{r u}$ are the distances $^{1}$ related to the $S$-LIS and LIS- $U$ links; $\tau$ stands for the path-loss exponent. $\theta_{n}$ and $\alpha_{n}$ are the adjustable phase shift and reflection coefficient of the $n$th LIS element. Thus, the corresponding SNR at $U$ can be expressed as

$$
\gamma=P_{S} \frac{\left|\sum_{n=1}^{N} g_{n} h_{n} d_{b r}^{-\frac{\tau}{2}} d_{r u}^{-\frac{\tau}{2}} \alpha_{n} e^{j \theta_{n}}\right|^{2}}{N_{0}}
$$

\footnotetext{
${ }^{1}$ Considering the fact that $S$ and $U$ are located at much more longer distances than the distances between the LIS elements, we assume that all the distances related to the individual elements are identical.
}

Recalling the perfect phase adjustment, in order to maximize the received SNR, the phase shifts can be optimized as [10]

$$
\begin{aligned}
\left(\theta_{1}^{o p t}, \ldots, \theta_{N}^{o p t}\right)=\left(-\left(\arg \left[g_{1}\right]\right.\right. & \left.+\arg \left[h_{1}\right]\right), \ldots, \\
& \left.-\left(\arg \left[g_{N}\right]+\arg \left[h_{N}\right]\right)\right),
\end{aligned}
$$

so that eventually each term of the summation will be cophased. Therefore, the optimal received SNR is found as

$$
\gamma=\frac{P_{S}}{N_{0}}\left(\sum_{n=1}^{N}\left|g_{n}\right|\left|h_{n}\right| \tilde{\gamma}_{n}\right)^{2}
$$

where $\tilde{\gamma}_{n}=\alpha_{n} d_{s r}^{-\frac{\tau}{2}} d_{r u}^{-\frac{\tau}{2}}$.

\section{PERFormance AnAlysis}

In this section, we study the OP, EC and BER using the abovementioned approximations for the considered LISassisted wireless system.

\section{A. Outage Performance}

Generally, the OP is defined as the probability that the achievable received SNR at the destination node falls below the predefined SNR-associated rate threshold $\gamma_{\mathrm{th}}$. To investigate the outage performance of the proposed system model, we rewrite $\gamma=\frac{P_{S} X^{2}}{N_{0}}$, where $X=\sum_{n=1}^{N} Y_{n} \tilde{\gamma}_{n}, Y_{n}=\left|g_{n}\right|\left|h_{n}\right|$, and $Y_{n}$ is subject to the double Nakagami- $m$ distribution.

1) CLT approximation: The CLT states that with a given sufficiently large number of passive LIS elements, the probability density function (PDF) and cumulative density function (CDF) of the sum of $N$ i.n.i.d. double Nakagami- $m$ RVs can be approximated by a Gaussian distribution as [14]

$$
\begin{aligned}
f_{X}^{\mathrm{CLT}}(x) & =\frac{\phi_{X}}{\sqrt{2 \pi \sigma_{X}^{2}}} \exp \left(-\frac{\left(x-\mu_{X}\right)^{2}}{2 \sigma_{X}^{2}}\right), \\
F_{X}^{\mathrm{CLT}}(x) & =1-\phi_{X} Q\left(\frac{x-\mu_{X}}{\sigma_{X}}\right),
\end{aligned}
$$

where $\phi_{X}=1 / Q\left(-\frac{\mu_{X}}{\sigma_{X}}\right)$ is the normalization coefficient, and $Q(\cdot)$ is the Gaussian $Q$ function, defined by [15, Eq. (6.287.3)]. The parameters $\mu_{X}$ and $\sigma_{X}^{2}$ are calculated as

$$
\begin{aligned}
& \mu_{X}=N \mathbb{E}\left[Y_{n}^{2}\right] \tilde{\gamma}_{n}, \\
& \sigma_{X}^{2}=N \sigma_{Y_{n}}^{2} \tilde{\gamma}_{n},
\end{aligned}
$$

where the $h$ th moment of $Y_{n}$ can be defined as [16]

$$
\mathbb{E}\left[Y_{n}^{h}\right]=\prod_{i=1}^{2} \frac{\Gamma\left(m_{i}+h / 2\right)}{\Gamma\left(m_{i}\right)}\left(\frac{\Omega_{i}}{m_{i}}\right)^{h / 2},
$$

where $m$ denotes the Nakagami- $m$ fading parameter, $\Gamma(t)=$ $\int_{0}^{\infty} x^{t-1} e^{-x} \mathrm{~d} x$ is the Gamma function.

The outage performance of the system can be defined as

$$
\begin{aligned}
F_{\gamma}(z) & =\operatorname{Pr}(\gamma<z) \\
& =\operatorname{Pr}\left(X<\sqrt{\frac{z}{\bar{\gamma}}}\right)=F_{X}\left(\sqrt{\frac{z}{\bar{\gamma}}}\right) .
\end{aligned}
$$


or equivalently, for the CLT approximation,

$$
F_{\gamma}^{\mathrm{CLT}}(z)=1-\phi_{X} Q\left(\frac{\sqrt{\frac{z}{\bar{\gamma}}}-\mu_{X}}{\sigma_{X}}\right)
$$

where $\bar{\gamma}=\frac{P_{S}}{N_{0}}$ is the transmit SNR. Thus, the OP can be obtained using its mathematical formulation as

$$
P_{\text {out }}^{\mathrm{CLT}}\left(\gamma_{\mathrm{th}}\right)=\operatorname{Pr}\left(\gamma<\gamma_{\mathrm{th}}\right)=F_{\gamma}^{\mathrm{CLT}}\left(\gamma_{\mathrm{th}}\right) \text {. }
$$

2) Gamma approximation: Another approach is to apply the Gamma approximation, whose PDF and CDF are given by

$$
\begin{aligned}
& f_{X}^{\Gamma}(x)=\frac{1}{\Gamma(l) \theta^{l}} x^{l-1} \exp \left(-\frac{x}{\theta}\right), \\
& F_{X}^{\Gamma}(x)=\frac{1}{\Gamma(l)} \gamma\left(l, \frac{x}{\theta}\right),
\end{aligned}
$$

where $\gamma(s, x)=\int_{0}^{x} t^{s-1} \exp (-t) \mathrm{d} t$ is the lower incomplete Gamma function; $l$ and $\theta$ are the shape and scale parameters calculated after equating the mean and variance of the Gamma distribution $\left(l \theta\right.$ and $\left.l \theta^{2}\right)$ to $\mu_{X}$ and $\sigma_{X}^{2}$, respectively. Similar to (10), the CDF of $\gamma$ is then written accordingly

$$
F_{\gamma}^{\Gamma}(z)=\frac{1}{\Gamma(l)} \gamma\left(l, \frac{\sqrt{z}}{\sqrt{\bar{\gamma}} \theta}\right) .
$$

Finally, the OP is defined as

$$
P_{\text {out }}^{\Gamma}\left(\gamma_{\mathrm{th}}\right)=F_{\gamma}^{\Gamma}\left(\gamma_{\mathrm{th}}\right)
$$

3) $K_{\mathrm{G}}$ approximation: In [12], the authors applied $K_{\mathrm{G}}$ distribution to approximate the sum of double-Rayleigh RVs by using the approach [17]. Due to the demonstrated tightness, we exploit to further extend the proposed method for the considered system model over double Nakagami- $m$ channels.

We first consider $G=\sum_{s=1}^{S} R_{s}^{2}$, where $R_{s}$ is a $K_{\mathrm{G}} \mathrm{RV}$. The author in [17] stated that the sum of $K_{\mathrm{G}}$ RVs can be tightly approximated by the PDF of $\sqrt{G}$, approximating $G$ with the squared $K_{\mathrm{G}}$ distribution. Then, the PDF of $X$ is expressed as

$$
f_{X}^{K_{\mathrm{G}}}(x)=\frac{4 \phi_{H}^{k_{w}+m_{w}} x^{k_{w}+m_{w}-1}}{\Gamma\left(m_{w}\right) \Gamma\left(k_{w}\right)} K_{k_{w}-m_{w}}\left(2 x \phi_{H}\right),
$$

where $K_{v}(\cdot)$ is the modified $v$ th-order Bessel function of the second kind [15, Eq. (8.432)], $\phi_{H}=\left(\frac{k_{w} m_{w}}{\Omega_{w}}\right)^{\frac{1}{2}}$, and $\Omega_{w}=$ $\mathbb{E}\left[X^{2}\right]$. The shaping parameters $m_{w}$ and $k_{w}$ are calculated as

$$
\begin{aligned}
m_{w} & =\frac{-\beta+\sqrt{\beta^{2}-4 a c}}{2 a}, \\
k_{w} & =\frac{-\beta-\sqrt{\beta^{2}-4 a c}}{2 a} .
\end{aligned}
$$

If $k_{w}$ and $m_{w}$ are conjugate complex numbers, they are set to the magnitude of the estimated complex parameters. The values of $\beta, a$, and $c$ are expressed as follows

$$
\begin{aligned}
& \beta=\mathbb{E}\left[X^{6}\right] \mathbb{E}\left[X^{2}\right]-4\left(\mathbb{E}\left[X^{4}\right]\right)^{2}+3\left(\mathbb{E}\left[X^{2}\right]\right)^{2} \mathbb{E}\left[X^{4}\right], \\
& a=\mathbb{E}\left[X^{6}\right] \mathbb{E}\left[X^{2}\right]+\left(\mathbb{E}\left[X^{2}\right]\right)^{2} \mathbb{E}\left[X^{4}\right]-2\left(\mathbb{E}\left[X^{4}\right]\right)^{2}, \\
& c=2\left(\mathbb{E}\left[X^{2}\right]\right)^{2} \mathbb{E}\left[X^{4}\right] .
\end{aligned}
$$

Using a multinomial identity and (9), these parameters are defined by evaluating the required moments of $X$ as

$$
\begin{aligned}
E\left[X^{n}\right]= & \sum_{n_{1}=0}^{n} \sum_{n_{2}=0}^{n_{1}} \cdots \sum_{n_{N-1}=0}^{n_{N-2}}\left(\begin{array}{c}
n \\
n_{1}
\end{array}\right)\left(\begin{array}{l}
n_{1} \\
n_{2}
\end{array}\right) \cdots\left(\begin{array}{c}
n_{N-2} \\
n_{N-1}
\end{array}\right) \\
& \times \mathbb{E}\left[Y_{1}^{n-n_{1}}\right] \mathbb{E}\left[Y_{2}^{n_{1}-n_{2}}\right] \cdots \mathbb{E}\left[Y_{N-1}^{n_{N-1}}\right] .
\end{aligned}
$$

Remark 1: The $h$ th moments of $X$ for the LIS-assisted wireless network are computationally complex, especially, for $h=\{4,6\}$. Therefore, to further facilitate the research in this area, they are calculated and drawn in Table I.

Finally, the PDF and CDF of $\gamma$ can be expressed as the squared $K_{\mathrm{G}}$ distribution as

$$
\begin{aligned}
f_{\gamma}^{K_{\mathrm{G}}}(z)= & \frac{2\left(\tilde{\gamma}_{n} \phi_{H}\right)^{k_{w}+m_{w}} z^{\frac{k_{w}+m_{w}}{2}}-1}{\bar{\gamma}^{\frac{k_{w}+m_{w}}{2}} \Gamma\left(m_{w}\right) \Gamma\left(k_{w}\right)} \\
& \times K_{k_{w}-m_{w}}\left(2 \tilde{\gamma}_{n} \phi_{H} \sqrt{\frac{z}{\bar{\gamma}}}\right), \\
F_{\gamma}^{K_{\mathrm{G}}}(z)= & \frac{G_{1,3}^{2,1}\left(\begin{array}{c}
1 \\
k_{w}, m_{w}, 0
\end{array} \mid \frac{z\left(\tilde{\gamma}_{n} \phi_{H}\right)^{2}}{\bar{\gamma}}\right)}{\Gamma\left(m_{w}\right) \Gamma\left(k_{w}\right)} .
\end{aligned}
$$

where $G_{p, q}^{m, n}\left(\begin{array}{c}a_{1}, \ldots, a_{p} \\ b_{1}, \ldots, b_{q}\end{array} \mid \eta x\right)$ is the Meijer G-function [15].

Remark 2: It is important to highlight that the direct application of a $K_{\mathrm{G}}$ distribution is characterized by insufficient accuracy for the case of LIS with large $N$.

Therefore, in this paper, we apply a Hankel's expansion that makes the $K_{\mathrm{G}}$ distribution highly accurate as [18]

$$
\begin{aligned}
f_{X}^{\mathrm{H}}(x) & =\frac{2 \phi_{\mathrm{H}} \exp \left(-2 \phi_{\mathrm{H}} x\right)}{\psi_{\mathrm{H}}} \sum_{s=0}^{M} b_{s}\left(2 \phi_{\mathrm{H}} x\right)^{\omega_{s}-1}, \\
F_{X}^{\mathrm{H}}(x) & =\frac{1}{\psi_{\mathrm{H}}} \sum_{s=0}^{M} b_{s} \gamma\left(\omega_{s}, 2 \phi_{\mathrm{H}} x\right),
\end{aligned}
$$

where $\omega_{s}=\mu_{\mathrm{H}}-s, b_{s}=\sum_{s=0}^{M} \frac{\Gamma\left(\frac{1}{2}+s\right)}{\Gamma\left(\frac{1}{2}-s\right) 2^{s}}, \mu_{\mathrm{H}}=m_{w}+k_{w}-\frac{1}{2}$, and $\psi_{\mathrm{H}}=\sum_{s=0}^{M} b_{s} \Gamma\left(\omega_{\mathrm{H}}\right) . M$ is defined as [18]

$$
M= \begin{cases}1, & \text { if } 0 \leq k_{w}-m_{w}<0.5 \\ \left\lceil k_{w}-m_{w}-0.5\right\rceil, & \text { if } k_{w}-m_{w} \geq 0.5\end{cases}
$$

Now, with the help of (23), (24) and (25), the PDF and CDF of $\gamma$ can be respectively described as

$$
\begin{aligned}
f_{\gamma}^{\mathrm{H}}(z)= & \frac{\phi_{\mathrm{H}} \exp \left(-2 \tilde{\gamma}_{n} \phi_{\mathrm{H}} \sqrt{\frac{z}{\bar{\gamma}}}\right)}{\psi_{\mathrm{H}}} \\
& \times \sum_{s=0}^{M} b_{s}\left(2 \phi_{\mathrm{H}}\right)^{\omega_{s}-1} \tilde{\gamma}_{n}^{\omega_{s}}\left(\frac{\sqrt{z}^{\omega_{s}-2}}{\sqrt{\bar{\gamma}}^{\omega_{s}}}\right), \\
F_{\gamma}^{\mathrm{H}}(z)= & \frac{1}{\psi_{\mathrm{H}}} \sum_{s=0}^{M} b_{s} \gamma\left(\omega_{s}, 2 \tilde{\gamma}_{n} \phi_{\mathrm{H}} \sqrt{\frac{z}{\bar{\gamma}}}\right) .
\end{aligned}
$$

Finally, the OP can be obtained as

$$
P_{\text {out }}^{\mathrm{H}}\left(\gamma_{\mathrm{th}}\right)=F_{\gamma}^{\mathrm{H}}\left(\gamma_{\mathrm{th}}\right) \text {. }
$$




\section{B. Ergodic Capacity}

1) CLT approximation: EC can be defined as [19]

$$
C=\mathbb{E}\left[\log _{2}(1+\gamma)\right] .
$$

Then, substituting $\gamma=\tilde{\gamma} x^{2}$, it can be rewritten as

$$
C=\frac{1}{\ln (2)} \int_{0}^{\infty} \ln \left(1+x^{2} \bar{\gamma}\right) f_{X}(x) \mathrm{d} x
$$

By inserting (5) into (30), we obtain

$$
C=\frac{1}{\ln (2)} \frac{\phi_{\mathrm{X}}}{\sqrt{2 \pi \sigma_{X}^{2}}} \int_{0}^{\infty} \ln \left(1+x^{2} \bar{\gamma}\right) \exp \left(-\frac{\left(x-\mu_{X}\right)^{2}}{2 \sigma_{X}^{2}}\right) \mathrm{d} x \text {. }
$$

Now, by using the curve fitting [20], $\ln \left(1+x^{2} \bar{\gamma}\right)$ can be well-approximated as $\sum_{i=1}^{4} \zeta_{i} \exp \left(\psi_{i} \bar{\gamma} x^{2}\right)$, where $\zeta=[6.4678,-1.8264,-2.7948,-1.6552]$ and $\psi=[0,0.037,0.004,0.274]$. Then, (31) can be rewritten as

$$
\begin{aligned}
C \approx \frac{1}{\ln (2)} & \frac{\phi_{\mathrm{X}}}{\sqrt{2 \pi \sigma_{X}^{2}}} \sum_{i=1}^{4} \\
& \times \int_{0}^{\infty} \zeta_{i} \exp \left(-\left(\eta_{i} x^{2}+2 \delta x+\rho\right)\right) \mathrm{d} x
\end{aligned}
$$

where $\eta_{i}=\frac{2 \psi_{i} \sigma_{X}^{2}+1}{2 \sigma_{X}^{2}}, \delta=-\frac{\mu_{X}}{2 \sigma_{X}^{2}}$, and $\rho=\frac{\mu_{X}^{2}}{2 \sigma_{X}^{2}}$. With the help of [15, Eq. (2.33.1)], the capacity can be written as

$$
\begin{aligned}
C^{\mathrm{CLT}} \approx \sum_{i=1}^{4} \frac{\zeta_{i} \phi_{\mathrm{X}}}{2 \ln (2) \sqrt{2 \eta_{i} \sigma_{X}^{2}}} & \exp \left(\frac{\delta^{2}-\eta_{i} \rho}{\eta_{i}}\right) \\
& \times\left(1-\operatorname{erf}\left(\frac{\delta}{\sqrt{\eta_{i}}}\right)\right),
\end{aligned}
$$

where $\operatorname{erf}(\cdot)$ indicates the error function [15, Eq. (8.250.1)].

2) Gamma approximation: Using the Gamma approximation, EC can be expressed as

$$
C=\frac{1}{\ln (2) \Gamma(l)} \int_{0}^{\infty} x^{l-1} \ln \left(1+x^{2} \bar{\gamma}\right) \exp \left(-\frac{x}{\theta}\right) \mathrm{d} x .
$$

According to [21], the natural logarithm and exponential function can be respectively transformed as

$$
\begin{aligned}
\ln \left(1+x^{2} \bar{\gamma}\right) & =G_{2,2}^{1,2}\left(\begin{array}{c}
1,1 \\
1,0
\end{array} \mid x^{2} \bar{\gamma}\right), \\
\exp \left(-\frac{x}{\theta}\right) & =G_{0,1}^{1,0}\left(\begin{array}{c|c}
- & x \\
0 & \bar{\theta}
\end{array}\right) .
\end{aligned}
$$

Further, by substituting (35) and (36) into (34) and using [21, Eq. (21)], we can rewrite the final closed-form EC for the Gamma distribution as

$$
\begin{aligned}
C^{\Gamma}= & \frac{1}{\ln (2) \Gamma(l) \theta^{l}} \int_{0}^{\infty} x^{l-1} G_{0,1}^{1,0}\left(\begin{array}{c|c}
- & x \\
0 & \bar{\theta}
\end{array}\right) \\
& \times G_{2,2}^{1,2}\left(\begin{array}{c}
1,1 \\
1,0
\end{array} \mid x^{2} \bar{\gamma}\right) \mathrm{d} x \\
= & \frac{2^{l-1}}{\ln (2) \Gamma(l) \sqrt{\pi}} G_{4,2}^{1,4}\left(\begin{array}{c}
1, \frac{1-l}{2}, \frac{2-l}{2}, 1 \\
1,0
\end{array} \mid 4 \theta^{2} \bar{\gamma}\right) .
\end{aligned}
$$

3) $K_{\mathrm{G}}$ approximation: Following (30), EC can be written as

$$
\begin{aligned}
C=\frac{\phi_{H} \exp \left(-2 \phi_{\mathrm{H}} x\right)}{\ln (2) \psi_{\mathrm{H}}} \sum_{s=0}^{M} \\
\quad \times \int_{0}^{\infty} \ln \left(1+\tilde{\gamma}_{n}^{4} x^{2} \bar{\gamma}\right) b_{s}\left(2 \phi_{\mathrm{H}} x\right)^{\omega_{s}-1} \mathrm{~d} x .
\end{aligned}
$$

Using (35) and (36), (38) can be expressed as

$$
\begin{aligned}
C=\frac{\phi_{\mathrm{H}}}{\ln (2) \psi_{\mathrm{H}}} \sum_{s=0}^{M} b_{s}\left(2 \phi_{\mathrm{H}}\right)^{\omega_{s}-1} \int_{0}^{\infty} x^{\omega_{s}-1} \\
\quad \times G_{2,2}^{1,2}\left(\begin{array}{c}
1,1 \\
1,0
\end{array} \mid \tilde{\gamma}_{n}^{4} x^{2} \bar{\gamma}\right) G_{0,1}^{1,0}\left(\begin{array}{c}
- \\
0
\end{array} 2 \phi_{\mathrm{H}} x\right) \mathrm{d} x .
\end{aligned}
$$

Finally, using [21, Eq. (21)], EC can be derived in closed-form as follows

$$
\begin{aligned}
& C^{\mathrm{H}}=\frac{\phi_{\mathrm{H}}}{\ln (2) \psi_{\mathrm{H}} \sqrt{\pi}} \sum_{s=0}^{M} b_{s} 2^{\omega_{s}-1} \\
& \times G_{4,2}^{1,4}\left(\begin{array}{c|c}
1, \frac{1-\omega_{s}}{2}, \frac{2-\omega_{s}}{2}, 1 & \frac{\tilde{\gamma}_{n}^{4} \bar{\gamma}}{\phi_{\mathrm{H}}^{2}}
\end{array}\right) .
\end{aligned}
$$

\section{Average BER}

The BER is one of the valuable performance metrics whose expression, considering different types of binary modulation schemes, is expressed as [16]

$$
P_{e}=\int_{0}^{\infty} p \operatorname{erfc}(\sqrt{q x}) f_{\gamma}(x) d x
$$

where $\operatorname{erfc}(\cdot)$ is complementary error function [15, Eq. (8.250.4)]. $p$ and $q$ are the parameters standing for various modulating schemes.

1) CLT approximation: By taking a derivative of (11), we derive the PDF of $\gamma$ as

$$
f_{\gamma}^{\mathrm{CLT}}(z)=\frac{\phi_{X}}{2 \sqrt{2 z \bar{\gamma} \pi \sigma_{X}^{2}}} \exp \left(-\frac{\left(\sqrt{\frac{z}{\bar{\gamma}}}-\mu_{X}\right)^{2}}{2 \sigma_{X}^{2}}\right) .
$$

Next, using (41) and (42), we evaluate the average BER as

$$
\begin{aligned}
P_{e}^{C L T}=\int_{0}^{\infty} \frac{p \phi_{X}}{2 \sqrt{2 x \bar{\gamma} \pi \sigma_{X}^{2}}} \exp \left(-\frac{\left(\sqrt{\frac{x}{\bar{\gamma}}}-\mu_{X}\right)^{2}}{2 \sigma_{X}^{2}}\right) \\
\times \operatorname{erfc}(\sqrt{q x}) \mathrm{d} x .
\end{aligned}
$$

For the best of authors' knowledge, the integral in (43) does not have a closed-form solution. Therefore, in this paper, the BER performance for the CLT approximation is evaluated numerically.

2) Gamma approximation: We first obtain the PDF of $\gamma$ by taking a derivative of (15) as

$$
f_{\gamma}^{\Gamma}(z)=\frac{z^{\frac{l}{2}-1}}{2 \Gamma(l) \sqrt{\bar{\gamma}^{l}}} \exp \left(-\frac{\sqrt{z}}{\sqrt{\bar{\gamma}} \theta}\right) .
$$


Table I. The $h$ th moments of $X$ for different $N$ and $m$ using (20).

\begin{tabular}{|c|c|c|c|c|}
\hline$N$ & $h$ & $m=1$ & $m=2$ & $m=3$ \\
\hline 32 & 4 & $460,212.89$ & $683,097.37$ & $785,686.33$ \\
& 6 & $347,089,602.76$ & $593,232,386.45$ & $719,172,506.96$ \\
\hline 64 & 4 & $6,869,200.92$ & $10,578,401.84$ & $12,306,711.07$ \\
& 6 & $19,029,952,117.34$ & $35,294,940,460.47$ & $43,890,709,549.17$ \\
\hline 96 & 4 & $33,951,087.97$ & $52,958,674.96$ & $61,853,300.08$ \\
& 6 & $205,395,599,531.19$ & $392,076,603,724.93$ & $491,879,380,153.50$ \\
\hline 128 & 4 & $106,005,701.76$ & $166,435,103.37$ & $194,774,768.18$ \\
& 6 & $1,122,862,585,680.33$ & $2,175,182,544,825.52$ & $2,741,103,413,827.63$ \\
\hline
\end{tabular}

The term erfc $(\sqrt{q x})$ can be represented using [21] as

$$
\operatorname{erfc}(\sqrt{q x})=\frac{1}{\sqrt{\pi}} G_{1,2}^{2,0}\left(\begin{array}{c|c}
1 & q x \\
0, \frac{1}{2} & q x
\end{array}\right) \text {. }
$$

Now, using (36), (41), (44) and (45), we evaluate the average BER for the Gamma approximation as

$$
\begin{aligned}
& P_{e}^{\Gamma}=\frac{p}{2 \Gamma(l) \sqrt{\bar{\gamma}^{l}}} \int_{0}^{\infty} x^{\frac{l}{2}-1} \operatorname{erfc}(\sqrt{q x}) \exp \left(-\frac{\sqrt{x}}{\sqrt{\bar{\gamma}} \theta}\right) \mathrm{d} x \\
& =\frac{p \bar{\gamma}^{\frac{-l}{2}}}{2 \Gamma(l) \sqrt{\pi}} \int_{0}^{\infty} x^{\frac{l}{2}-1} G_{0,1}^{1,0}\left(\begin{array}{c|c}
- & \frac{\sqrt{x}}{0}
\end{array}\right) G_{1,2}^{2,0}\left(\begin{array}{c}
1 \\
0, \frac{1}{2}
\end{array} \mid q x\right) \mathrm{d} x \\
& =\frac{p}{2 \pi \Gamma(l)}\left(\frac{q}{\bar{\gamma} \theta^{2}}\right)^{\frac{l}{2}} G_{2,3}^{2,2}\left(\begin{array}{c|c}
\frac{1}{2}-\frac{l}{2}, 1-\frac{l}{2} & \frac{1}{4, \frac{1}{2},-\frac{l}{2}}
\end{array}\right) \text {. }
\end{aligned}
$$

3) $K_{\mathrm{G}}$ approximation: Using (26) and (41), the BER for the Hankel-based $K_{\mathrm{G}}$ approximation can be written as

$$
\begin{aligned}
P_{e}^{\mathrm{H}}= & \frac{p\left(2 \phi_{\mathrm{H}}\right)^{\omega_{\mathrm{H}}}}{2 \psi_{\mathrm{H}}} \sum_{s=0}^{M} \frac{b_{s}}{\sqrt{\bar{\gamma}^{\omega_{s}}}} \\
& \times \int_{0}^{\infty} \operatorname{erfc}(\sqrt{q x}) x^{\frac{\omega_{s}}{2}}-1 \exp \left(-\frac{2 \phi_{\mathrm{H}}}{\tilde{\gamma}_{n}^{2}} \sqrt{\frac{x}{\bar{\gamma}}}\right) \mathrm{d} x \\
= & \frac{p\left(2 \phi_{\mathrm{H}}\right)^{\omega_{\mathrm{H}}}}{2 \pi \psi_{\mathrm{H}}} \sum_{s=0}^{M} b_{s}\left(\frac{\tilde{\gamma}_{n}^{2}}{q \bar{\gamma}}\right)^{\frac{\omega_{s}}{2}} \\
& \times G_{2,3}^{2,2}\left(\begin{array}{c}
1-\frac{\omega_{s}}{2}, \frac{1}{2}-\frac{\omega_{s}}{2} \\
0, \frac{1}{2},-\frac{\omega_{s}^{2}}{2}
\end{array} \frac{\phi_{\mathrm{H}}^{2}}{q \tilde{\gamma}_{n}^{4} \bar{\gamma}}\right) .
\end{aligned}
$$

\section{NumericAl RESUlts AND Discussion}

In this section, we present some analytical and simulation results on the performance of a LIS-enabled wireless system. Unless stated otherwise, for the simulation purposes, the following parameters are considered: $m=2, \alpha_{n}=1, d_{s r}=d_{r u}=10$ $\mathrm{m}, \tau=2, \gamma_{\mathrm{th}}=5 \mathrm{~dB}$, and $N_{0}=1$. Also, we use quadrature phase shift keying (QPSK) with $p=0.5$ and $q=0.5$.

Fig. 2 illustrates the system OP for a different number of LIS elements. Both Gamma and $K_{\mathrm{G}}$ approximations are fairly accurate for the whole range of transmit SNR values and different $N$, while the CLT one fits well with the simulation results only given considerably large $N$. One can notice outage performance improvement as the number of $N$ increases due to achievable high passive beamforming gains. From this perspective, it can be concluded that the increase in $N$ supports the system's energy efficiency performance.

Fig. 3 depicts the EC results plotted versus the transmit SNR

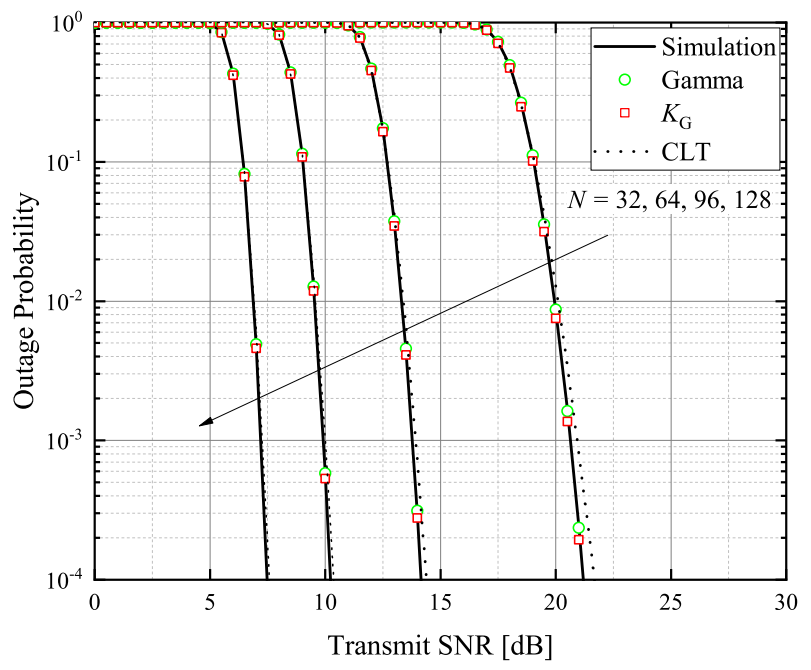

Fig. 2. OP vs. transmit SNR for different $N$.

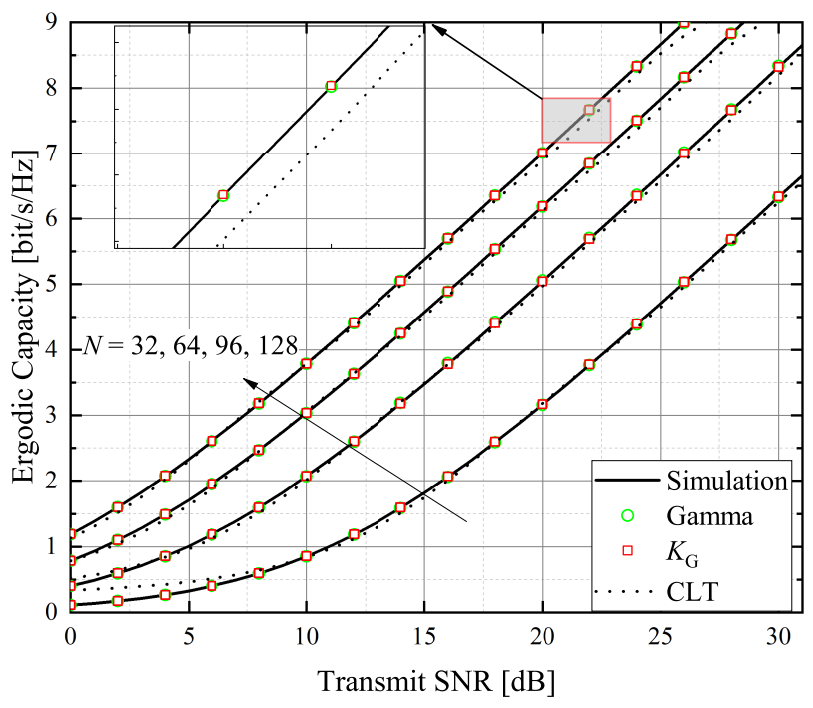

Fig. 3. EC vs. transmit SNR for different $N$.

for different values of $N$. The analytical expressions derived for the Gamma and $K_{\mathrm{G}}$ approximations are highly consistent with the simulation results compared to the CLT one. Moreover, one can notice that given linear increase in the number of $N$ does not result in a pro-rata gain in the capacity performance. For example, at $\bar{\gamma}=20 \mathrm{~dB}$, as $N$ increases from 32 to 64 , the EC 


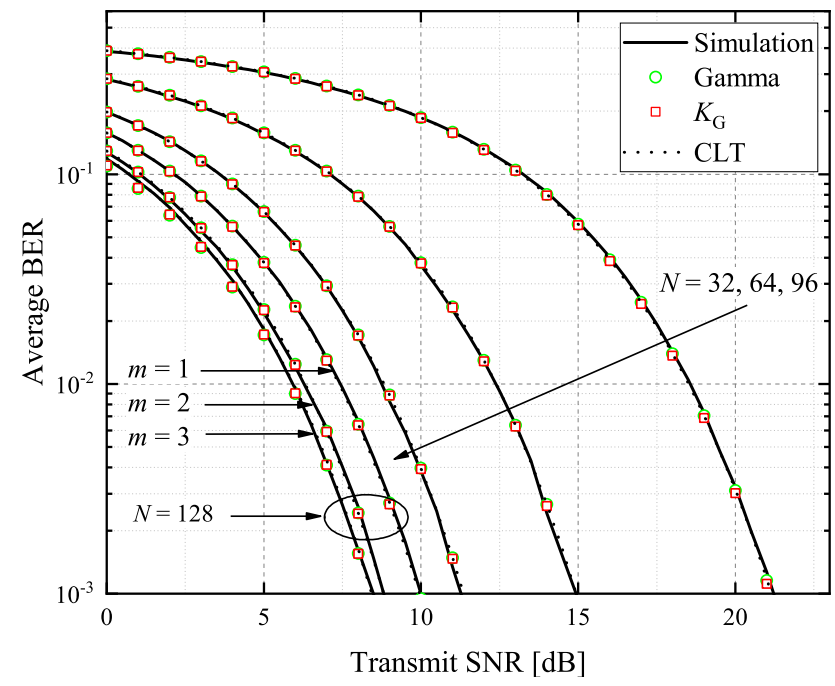

Fig. 4. Average BER vs. transmit SNR for different $N$.

improves by about $76.3 \%$, while, when $N$ goes up from 64 to 96 , the capacity rises by approximately $47.3 \%$.

Finally, the average BER performance is plotted in Fig. 4, considering the QPSK modulation for $N=32,64,96,128$ and $m=1,2,3$. When $m=1$, the considered fading model becomes double Rayleigh. It can be seen that bigger $m$ corresponds to the better system performance as $m$ stands for the number of multi-path components of the channel links. Moreover, the increase in $N$ significantly enhances the BER performance, i.e., for $m=2, N=32,64,96,128$ result in the transmit SNR of 21, 15, 11.2 and $8.5 \mathrm{~dB}$ required to achieve $10^{-3}$. However, this improvement has a non-linear nature that brings us to the conclusion that, for the considered system model, it is not always required to have a complicated LISbased relay as more densely deployed LIS leads to a higher amount of phase-shift errors.

\section{CONCLUSION}

The present work investigated the performance of the considered LIS-enabled system in terms of the OP, EC, and BER metrics considering different approximation methods. Closedform expressions for the given performance metrics were derived. The results revealed that both Gamma and $K_{\mathrm{G}}$ perform better than the CLT. Even though both Gamma and $K_{\mathrm{G}}$ wellapproximated the simulation results, the Gamma was proved to be less complicated in terms of the PDF and CDF representations, and, as a result, can be considered to be more practical. It also was shown that the fading parameter $m$ improves the system BER performance. Finally, the system performance mainly depends on the number of elements implemented.

\section{ACKNOWLEDGEMENT}

This work was supported by the Nazarbayev University Faculty Development Competitive Research Program under Grant no. 240919FD3935.

\section{REFERENCES}

[1] Q. Wu and R. Zhang, "Towards smart and reconfigurable environment: Intelligent reflecting surface aided wireless network," IEEE Communications Magazine, vol. 58, no. 1, pp. 106-112, 2019.

[2] S. Hu, F. Rusek, and O. Edfors, "Beyond massive MIMO: The potential of data transmission with large intelligent surfaces," IEEE Transactions on Signal Processing, vol. 66, no. 10, pp. 2746-2758, 2018.

[3] Z.-Q. He and X. Yuan, "Cascaded channel estimation for large intelligent metasurface assisted massive MIMO," IEEE Wireless Communications Letters, vol. 9, no. 2, pp. 210-214, 2019.

[4] Y. Liu, X. Liu, X. Mu, T. Hou, J. Xu, M. Di Renzo, and N. Al-Dhahir, "Reconfigurable intelligent surfaces: Principles and opportunities," IEEE Communications Surveys \& Tutorials, 2021.

[5] E. Björnson, Ö. Özdogan, and E. G. Larsson, "Intelligent reflecting surface versus decode-and-forward: How large surfaces are needed to beat relaying?," IEEE Wireless Communications Letters, vol. 9, no. 2, pp. 244-248, 2019.

[6] Y.-C. Liang, R. Long, Q. Zhang, J. Chen, H. V. Cheng, and H. Guo, "Large intelligent surface/antennas (lisa): Making reflective radios smart," Journal of Communications and Information Networks, vol. 4, no. 2, pp. 40-50, 2019.

[7] Q. Wu and R. Zhang, "Beamforming optimization for wireless network aided by intelligent reflecting surface with discrete phase shifts," IEEE Transactions on Communications, vol. 68, no. 3, pp. 1838-1851, 2019.

[8] T. Hou, Y. Liu, Z. Song, X. Sun, Y. Chen, and L. Hanzo, "Reconfigurable intelligent surface aided noma networks," IEEE Journal on Selected Areas in Communications, vol. 38, no. 11, pp. 2575-2588, 2020.

[9] L. Bariah, S. Muhaidat, P. C. Sofotasios, F. E. Bouanani, O. A. Dobre, and W. Hamouda, "Large intelligent surface-assisted nonorthogonal multiple access for $6 \mathrm{~g}$ networks: Performance analysis," IEEE Internet of Things Journal, vol. 8, no. 7, pp. 5129-5140, 2021.

[10] D. Kudathanthirige, D. Gunasinghe, and G. Amarasuriya, "Performance analysis of intelligent reflective surfaces for wireless communication," in IEEE International Conference on Communications (ICC), pp. 1-6, IEEE, 2020.

[11] N. K. Kundu and M. R. McKay, "RIS-assisted MISO communication: Optimal beamformers and performance analysis," in 2020 IEEE Globecom Workshops (GC Wkshps), pp. 1-6, 2020.

[12] L. Yang, F. Meng, Q. Wu, D. B. da Costa, and M.-S. Alouini, "Accurate closed-form approximations to channel distributions of RIS-aided wireless systems," IEEE Wireless Communications Letters, vol. 9, no. 11, pp. 1985-1989, 2020.

[13] E. Basar, M. Di Renzo, J. De Rosny, M. Debbah, M.-S. Alouini, and R. Zhang, "Wireless communications through reconfigurable intelligent surfaces," IEEE Access, vol. 7, pp. 116753-116773, 2019.

[14] J. Proakis, "Digital communications," McGraw-Hill, pp. 777-778, 2001.

[15] A. Jeffrey and D. Zwillinger, Table of integrals, series, and products. Elsevier, 2007.

[16] G. K. Karagiannidis, N. C. Sagias, and P. T. Mathiopoulos, " $N$ Nakagami: A novel stochastic model for cascaded fading channels," IEEE Transactions on Communications, vol. 55, no. 8, pp. 1453-1458, 2007.

[17] K. P. Peppas, "Accurate closed-form approximations to generalised- $K$ sum distributions and applications in the performance analysis of equalgain combining receivers," IET communications, vol. 5, no. 7, pp. 982989, 2011.

[18] H. Fu, S. Roy, and J. Cheng, "Applying Hankel's expansion for performance analysis in double-Nakagami (generalized- $K$ ) fading channels," IEEE Transactions on Communications, vol. 66, no. 10, pp. 4893-4906, 2018.

[19] A. J. Goldsmith and P. P. Varaiya, "Capacity of fading channels with channel side information," IEEE Transactions on Information Theory, vol. 43, no. 6, pp. 1986-1992, 1997.

[20] E. Salahat and A. Hakam, "Novel unified expressions for error rates and ergodic channel capacity analysis over generalized fading subject to AWGGN," in 2014 IEEE Global Communications Conference, pp. 39763982, IEEE, 2014.

[21] V. Adamchik and O. Marichev, "The algorithm for calculating integrals of hypergeometric type functions and its realization in REDUCE system," in Proceedings of the international symposium on Symbolic and algebraic computation, pp. 212-224, 1990. 